\title{
Iron 1s X-ray photoemission of $\mathrm{Fe}_{2} \mathrm{O}_{3}$
}

\author{
P.S. Miedema ${ }^{\mathrm{a}, *}$, F. Borgatti $^{\mathrm{b}}$, F. Offic $^{\mathrm{c}}$, G. Panaccione ${ }^{\mathrm{d}}$, F.M.F. de Groot ${ }^{\mathrm{a}}$ \\ a Inorganic Chemistry and Catalysis, Debye Institute for Nanomaterials Science, Utrecht University, Universiteitsweg 99,3584 CG Utrecht, The Netherlands \\ b CNR-ISMN, Instituto per Io Studio di Materiali Nanostrutturati, Via Gobetti 101, I-40129 Bologna, Italy \\ c Dipartimento di Scienze, Università di Roma Tre, I-00146 Rome, Italy \\ d Consiglio Nazionale delle Ricerche, CNR-IOM, Laboratorio TASC, Area Science Park, I-34149 Trieste, Italy
}

\section{A R T I C L E I N F O}

\section{Article history:}

Received 16 March 2015

Received in revised form 1 May 2015

Accepted 4 May 2015

Available online 12 May 2015

\section{Keywords:}

X-ray photoemission

HAXPES

Transition metal oxides

\begin{abstract}
A B S T R A C T
We present the 1s X-ray photoemission spectrum of $\alpha-\mathrm{Fe}_{2} \mathrm{O}_{3}$ in comparison with its $2 \mathrm{p}$ photoemission spectrum. We show that in case of transition metal oxides, because the $1 \mathrm{~s}$ core hole is not affected by core hole spin-orbit coupling and almost not affected by core-valence multiplet effects, the Fe $1 \mathrm{~s}$ spectrum and the complementary charge transfer multiplet calculations allow for an accurate determination of the charge transfer parameters. The consistency of the obtained parameters for the 1 s photoemission was confirmed with $2 \mathrm{p}$ photoemission calculations and compared to $2 \mathrm{p}$ experimental photoemission spectra.
\end{abstract}

(C) 2015 Elsevier B.V. All rights reserved.

\section{Introduction}

Hard X-ray photoemission (HAXPES), a technique with broad possibilities for studies of complex materials and buried-layer nanostructures, is photoemission with excitation energies ranging from $\sim 1.5 \mathrm{keV}$ to more than $10 \mathrm{keV}$ [1]. Due to the increased electron inelastic mean free paths of the photoelectrons using these large excitation energies, it is possible to apply photoemission to probe "true" bulk composition, atomic structure and electronic structure, as well as depth profiling for many systems of interest ranging from single compounds to multilayer nanostructures. In addition to the possibility of measuring more bulk-sensitive spectra for buried regions and interfaces, HAXPES allows to promote the emission of photoelectrons from core levels deeper than those usually accessible with conventional soft X-ray sources.

In particular for 3d transition metal (TMs) elements the common choice for photoemission studies concern mostly the 2 p and 3 s core level spectra, whose binding energies are in between 400 and $1000 \mathrm{eV}$, are easily accessible by employing quasi-monochromatic radiation provided by X-ray laboratory $(\mathrm{Mg} \mathrm{K} \alpha$ and $\mathrm{Al} \mathrm{K} \alpha$ at

\footnotetext{
* Corresponding author. Current address: Institute for Methods and Instrumentation in Synchrotron Radiation Research FG-ISRR, Helmholtz-Zentrum für Materialien und Energie GmbH, Albert-Einstein-Strasse 15, 12489 Berlin, Germany. Tel.: +4930 806214821 .

E-mail address: p.s.miedema@gmail.com (P.S. Miedema).
}

$1253.4 \mathrm{eV}$ and $1486.3 \mathrm{eV}$, respectively) or synchrotron radiation sources to access the whole soft X-ray range. The $2 \mathrm{p}$ or $3 \mathrm{~s}$ core level wavefunction overlaps strongly with the $3 \mathrm{~d}$ states and make these $2 \mathrm{p}$ and $3 \mathrm{~s}$ spectra sensitive to the electronic and magnetic structure of the probed elements [2] and in many cases the interpretation of the spectra has been well-established using the charge transfer multiplet model [3-6].

However, using synchrotron radiation with energy larger than $1.5 \mathrm{keV}$ or a X-ray source equipped with $\mathrm{Cu}$, Ti or $\mathrm{Cr}$ anodes [7-9], deeper core levels of the TMs can be studied, concerning in particular the measurement of the photoelectron emission from $1 \mathrm{~s}$ levels and the Auger transitions associated with the formation of a 1 s core hole [10-12]. HAXPES of 1s core levels was performed just for a few systems concerning iron metal and other $3 \mathrm{~d}$ transition metals before, using a $\mathrm{Cu} \mathrm{K} \alpha_{1}$ source [7,13], and on Ti metal and $\mathrm{TiO}_{2}$ $[14,15]$ or using synchrotron radiation on $\mathrm{Ni}$ metal $[16]$ and nickel oxide [17] and Mn 1s photoemission of MnAs and $\mathrm{Bi}_{1.91} \mathrm{Mn}_{0.09} \mathrm{Te}$ [18]. Apart for these sparse measurements, no specific attempt focusing explicitly on the amount of information that could be obtained through the theoretical analysis of $1 \mathrm{~s}$ core level spectra of TMs compounds has been performed up to now. To this purpose, here we present the study of the Fe 1s HAXPES spectrum for $\alpha-\mathrm{Fe}_{2} \mathrm{O}_{3}$.

The $1 \mathrm{~s}$ photoemission structure of $\mathrm{Fe}_{2} \mathrm{O}_{3}$ is expected to possess a relatively simple spectral shape due to the absence of core spinorbit splitting and due to negligible 1 s exchange splitting due to the small overlap between $1 \mathrm{~s}$ and unfilled 3d orbitals [4]. Also the relative binding energy differences between different oxidation states 
are expected to be larger, allowing more straightforward oxidation state determination. This implies that the Fe $1 \mathrm{~s}$ spectrum is not affected by core hole spin-orbit coupling and also it is not affected by core hole-valence multiplet effects, in contrast to the 2 p photoemission spectral shape. Although these multiplet effects are useful for symmetry and spin determination in X-ray absorption $[5,19]$, they complicate the detailed analysis of $2 \mathrm{p}$, 3s and $3 \mathrm{p}$ photoemission spectra with regard to charge transfer parameters. In other words, the 1s metal spectra might turn out to be ideal spectra for the determination of the charge transfer parameters without the complications of additional effects. In addition, the charge transfer parameters determined from $1 \mathrm{~s}$ photoemission, might serve well in resonant Auger analysis. The consistency of this analysis is confirmed through the good agreement occurring for calculating also the Fe $2 p$ spectrum with the same values of the charge transfer parameters. To our knowledge Fe 1s photoemission has not been applied on iron oxides before and in addition, the $1 \mathrm{~s}$ photoemission have not been calculated quantitatively before.

\section{Experiment}

Hard X-ray photoemission spectroscopy was performed on an $\alpha-\mathrm{Fe}_{2} \mathrm{O}_{3}$ single crystal with photon energies of $10 \mathrm{keV}$ and $7.7 \mathrm{keV}$ for the Fe $1 \mathrm{~s}$ and the Fe 2 p spectra respectively. No surface preparation was performed prior to the experiment. The incidence angle of the X-ray beam was set at 45 degrees, while the emitted photoelectrons were collected at normal emission geometry. According to the Tanuma Powell and Penn algorithm (TPP-2M) formula $[20,21]$ the inelastic electron mean free path (IMFP) for the $2 \mathrm{p}(1 \mathrm{~s})$ photoemission experiments with kinetic energy of about 7 (2.9) $\mathrm{keV}$ is 9.5 (4.6) $\mathrm{nm}$. Accordingly, the information depth, roughly estimated as three times the IMFP was about $30 \mathrm{~nm}$ and $13 \mathrm{~nm}$ for the Fe 2p photoemission and Fe 1s photoemission, respectively, thus ensuring the bulk sensitivity typical of HAXPES measurements. The experiments were performed with the VOLPE spectrometer on beamline ID16 of ESRF [22,23], with an overall resolution of $0.48 \mathrm{eV}$ at $10 \mathrm{keV}$ and $0.42 \mathrm{eV}$ at $7.7 \mathrm{keV}$. The energy scale is aligned on the Fermi energy or the $3 \mathrm{~d}$ core level of a gold foil. All the measurements on the sample were collected at room temperature.

\section{Theory}

The photoemission spectra were calculated using the charge transfer multiplet program [5], in which a number of correlated ionic configurations together generate the ground state. The charge transfer multiplet program is based on Cowan's atomic code [24] and extended by Theo Thole et al. to implement symmetry [25] and charge transfer $[5,26,27]$. We limited the number of ionic configurations (EGx) to three and describe the ground state of $\alpha-\mathrm{Fe}_{2} \mathrm{O}_{3}$ with respectively EG1 $=3 d^{5}, E G 2=3 d^{6} \underline{L}$ and EG3 $=3 d^{7} \underline{L L^{\prime}}$, where $\underline{L}$ indicates an oxygen $2 \mathrm{p}$ hole. Note that this means that the iron system is described by some combination of the three ionic configurations based on the energy differences, with detailed description below, between these three configurations. The energies of these three ionic states depend on the charge transfer parameters $\Delta, U$ and $Q$ as shown in Fig. 1. These parameters have the following physical meaning: $\Delta$ is the charge transfer energy related to the energy difference between $3 \mathrm{~d}^{5}$ and $3 \mathrm{~d}^{6} \underline{\mathrm{L}}$. $U$ is the Hubbard $U$ parameter, the $3 d-3 d$ Coulomb interaction potential and identifies with the energy difference of $3 \mathrm{~d}^{n}+3 \mathrm{~d}^{n}$ and $3 \mathrm{~d}^{n-1}+3 \mathrm{~d}^{n+1}$. $Q$ is the core hole potential. We assumed that the core hole potential is the same for the $1 \mathrm{~s}$ and the $2 \mathrm{p}$ core holes.

Although we only implemented three ionic configurations, we show the fourth ionic configuration as well in Fig. 1, in order to point out how much higher in energy this fourth configuration in both the initial and final state (EG4 and EF4) would be. In general, the relative energy of EG4 and EF4 shows that this fourth configuration can often be neglected.

The mixing of the ionic configurations through the interaction with the oxygen ligands was done with so-called hopping matrices $T$. This is an interaction of the $3 d$ states with a ligand p state, meaning that the hopping matrices are interactions between the oxygen ligand $2 \mathrm{p}$ state and the different $e_{\mathrm{g}}$ and $t_{2 \mathrm{~g}} 3 \mathrm{~d}$-states of the iron in octahedral symmetry. We use $T e_{\mathrm{g}}=2 * T t_{2 \mathrm{~g}}$ in our calculations [28]. Thus, effectively these hopping matrices $T \mathrm{e}_{\mathrm{g}}$ and $T t_{2 g}$ allow the interaction of the different ionic configurations with each other.

In addition to the fact that three ionic configurations were mixed, we simulated octahedral symmetry with a semi-empirical chosen crystal field energy (10Dq) of $1.9 \mathrm{eV}$ and for all the three

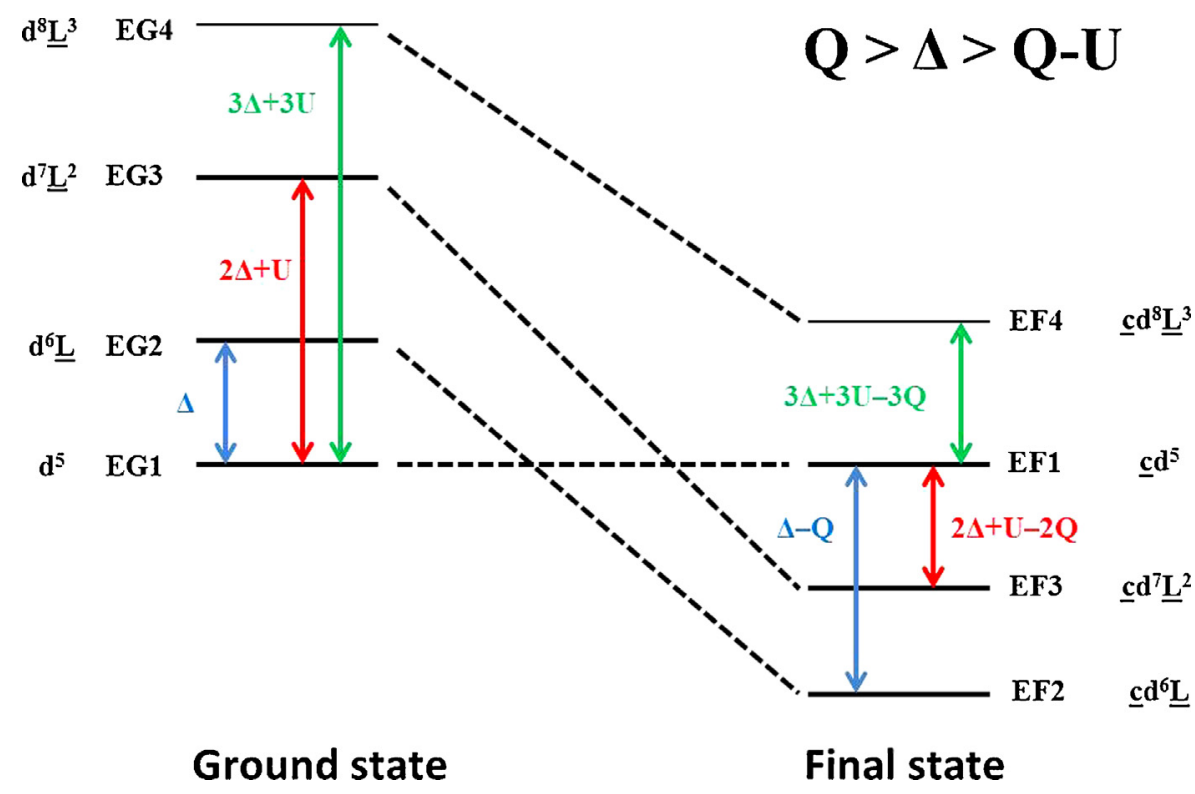

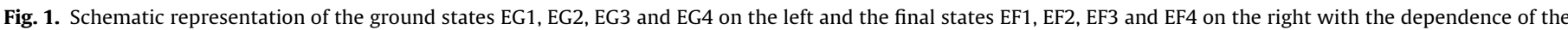

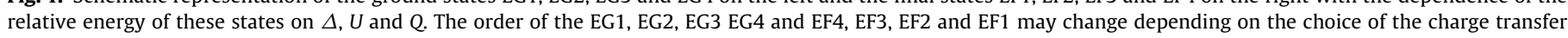
parameters, but the present order validity is stressed with the equation at the right top. 
separate configurations, electron correlations are taken into account with the Slater integrals $\mathrm{F}^{\mathrm{dd}}$ and $\mathrm{F}^{\mathrm{sd}}$ and $\mathrm{G}^{\text {sd }}$ (for $1 \mathrm{~s}$ photoemission, for $2 \mathrm{p}$ photoemission the last two are $\mathrm{F}^{\mathrm{pd}}$ and $\mathrm{G}^{\mathrm{pd}}$ ). The Hartree-Fock calculated Slater integral values are reduced to 85\% to overcome Hartree-Fock errors and to compensate slightly for covalence [5]. We note that the $\mathrm{F}^{\mathrm{dd}}$ for $1 \mathrm{~s}$ photoemission and $2 p$ photoemission is the same, while both $\mathrm{F}^{\text {sd }}$ and $\mathrm{G}^{\text {sd }}$ are orders of magnitude smaller than the $\mathrm{F}^{\mathrm{pd}}$ and $\mathrm{G}^{\mathrm{pd}}$ respectively, signifying that the $1 \mathrm{~s}$ photoemission is not perturbed much by multiplet interactions.

The results of the charge transfer multiplet calculations, the calculated transition energies and the corresponding oscillator strengths (sticks) were broadened with a Lorentzian and a Gaussian to account for the life time broadening and experimental resolution respectively.

\section{Results and discussion}

Fig. 2 shows the experimental Fe $2 \mathrm{p}$ (A) and $1 \mathrm{~s}$ (B) HAXPES spectra of $\mathrm{Fe}_{2} \mathrm{O}_{3}$. The $2 \mathrm{p}$ HAXPES spectrum shows two main peaks split by the $2 \mathrm{p}$ spin-orbit coupling indicated with $2 \mathrm{p}_{3 / 2}$ and $2 \mathrm{p}_{1 / 2}$ respectively plus two clear satellite structures $S$ and $S^{\prime}$ that have been assigned to charge transfer excitations. The relative energies of $S$, $2 \mathrm{p}_{1 / 2}$ and $\mathrm{S}^{\prime}$ relative to $2 \mathrm{p}_{3 / 2}$ are $8.37,13.76$ and $22.5 \mathrm{eV}$ respectively. In the following we compare the Fe $2 \mathrm{p}$ photoemission spectrum with reference literature, but first we have a look at the Fe 1s HAXPES. The excitation of a $1 \mathrm{~s}$ electron implies that the final state is not affected by spin-orbit coupling and also not by strong core-hole
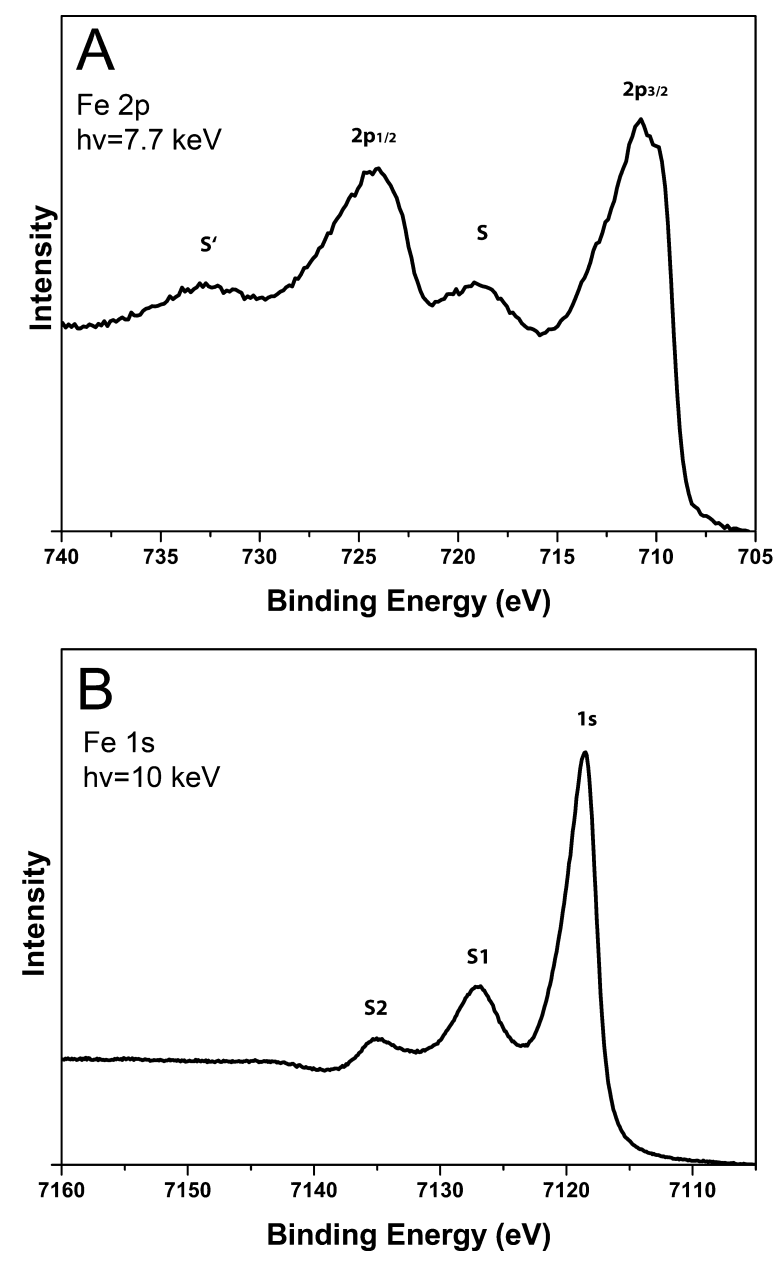

Fig. 2. Fe $2 p(A)$ and Fe $1 \mathrm{~s}(\mathrm{~B}) \mathrm{HAXPES}$ spectra of $\alpha-\mathrm{Fe}_{2} \mathrm{O}_{3}$. induced multiplet effects, however the 1 s photoemission spectrum shown in Fig. 2B shows three peaks named 1s, S1 and S2 where S1 and S2 are mainly due to charge transfer effects, as will be analyzed below. The relative energy of S1 and S2 to the main 1s peak is 8.57 and $16.56 \mathrm{eV}$ respectively. Note that the Fe $1 \mathrm{~s}$ HAXPES spectrum shown in Fig. $2 \mathrm{~B}$ is the first Fe $1 \mathrm{~s}$ spectrum of $\mathrm{Fe}_{2} \mathrm{O}_{3}$ to our knowledge.

We continue with the analysis of the more common $2 \mathrm{p}$ photoemission, since there are charge transfer parameters known for simulation of the spectrum and as well there are reference spectra available. The Fe $2 p$ photoemission spectrum of $\mathrm{Fe}_{2} \mathrm{O}_{3}$ has been studied before in combination with detailed multiplet analysis [29-31]. Fujii et al. have shown an $\mathrm{Fe}_{2} \mathrm{O}_{3}$ photoemission spectrum combined with a charge transfer multiplet calculation using two configurations $\left(\mathrm{Fe}^{3+}, \mathrm{d}^{5}\right.$ and $\left.\mathrm{d}^{6} \underline{\mathrm{L}}\right)$ with $\Delta=2 \mathrm{eV}, T e_{\mathrm{g}}=2.6\left(\mathrm{Tt}_{2 \mathrm{~g}}=1.3\right)$, $U=7.5 \mathrm{eV}$ and $Q=8.0 \mathrm{eV}$ and a crystal field energy of $10 \mathrm{Dq}=0.5 \mathrm{eV}$ [31] (see also Table 1). Their crystal field value $10 \mathrm{Dq}$ of $0.5 \mathrm{eV}$ is (too) low for $\mathrm{Fe}^{3+}$ and a value of 10Dq of approximately $1.5 \mathrm{eV}$ would be more in line with $2 \mathrm{p} \mathrm{X}$-ray absorption analysis [5,32], but we note that the exact value of $10 \mathrm{Dq}$ is less important for the shape of the $2 \mathrm{p}$ photoemission spectrum that is dominated by 2 p spin-orbit coupling and charge transfer effects. We use these Fujii et al. charge transfer parameters, defined as set B, as a starting point for our fits. It has been found that simulations improve if three electronic configurations are used for the simulation of $2 \mathrm{p}$ photoemission spectra [33].

Our Fe 2p HAXPES spectrum resembles the soft X-ray 2p Xray photoemission of $\mathrm{Fe}_{2} \mathrm{O}_{3}$ from literature [29,31,34-36] as can be confirmed from Fig. $3 \mathrm{~A}$ in comparison to two reference spectra [31,34]. The differences between the reference experimental 2p photoemission spectra of Fujii et al. [31] and Droubay et al. [34] are in the 708-710 eV binding energy (BE) region. The doublepeak-like pattern in this region of the photoemission measured at normal emission $\left(90^{\circ}\right)$ published by Droubay et al. is different from the observation of a single broader peak with a shoulder at lower BE in the spectrum of Fujii et al. [31]. This difference was ascribed to the difference in geometric and electronic structure of the Fe at the surface relative to the bulk [34]. In short, since there is a bulk contribution with different symmetry than the surface (with lower symmetry), there is a slight energy difference between the two components. In the bulk 2p HAXPES spectrum measured by us also two contributions at the main peak at $710 \mathrm{eV}$ binding energy are encountered (Fig. 3A, black line): the peak at lower $\mathrm{BE}$ is attributed to surface related photoemission and the lower

Table 1

Energy of the ground states (EG1, EG2 and EG3) and final states (EF1, EF2, EF3) for two sets $\mathrm{A}$ and $\mathrm{B}$ of $\Delta, U$ and $Q$ for analysis of $\mathrm{Fe}_{2} \mathrm{O}_{3}$. The relative ground state contributions (\% EG1, \%EG2 and \%EG3) in the photoemission calculation are presented as well.

\begin{tabular}{lcc}
\hline Set & $\mathrm{A}$ & $\mathrm{B}[31]$ \\
\hline$\Delta$ & 3.0 & 2.0 \\
$U$ & 7.5 & 7.5 \\
$Q$ & 9.0 & 8.0 \\
EG1 & 0 & 0 \\
EG2 & 3.0 & 2.0 \\
EG3 & 13.5 & 11.5 \\
EG4 & 31.5 & 28.5 \\
10 Dq & 1.9 & 0.5 \\
EF1 & 0 & 0 \\
EF2 & -6.0 & -6.0 \\
EF3 & -4.5 & -4.5 \\
EF4 & 4.5 & 4.5 \\
$\%$ EG1 & 54.1 & 48.3 \\
$\%$ EG2 & 41.7 & 46.6 \\
$\%$ EG3 & 4.2 & 5.1 \\
$T e_{g}$ & 2.7 & 2.6 \\
$T t_{2 g}$ & 1.35 & 1.3 \\
\hline
\end{tabular}




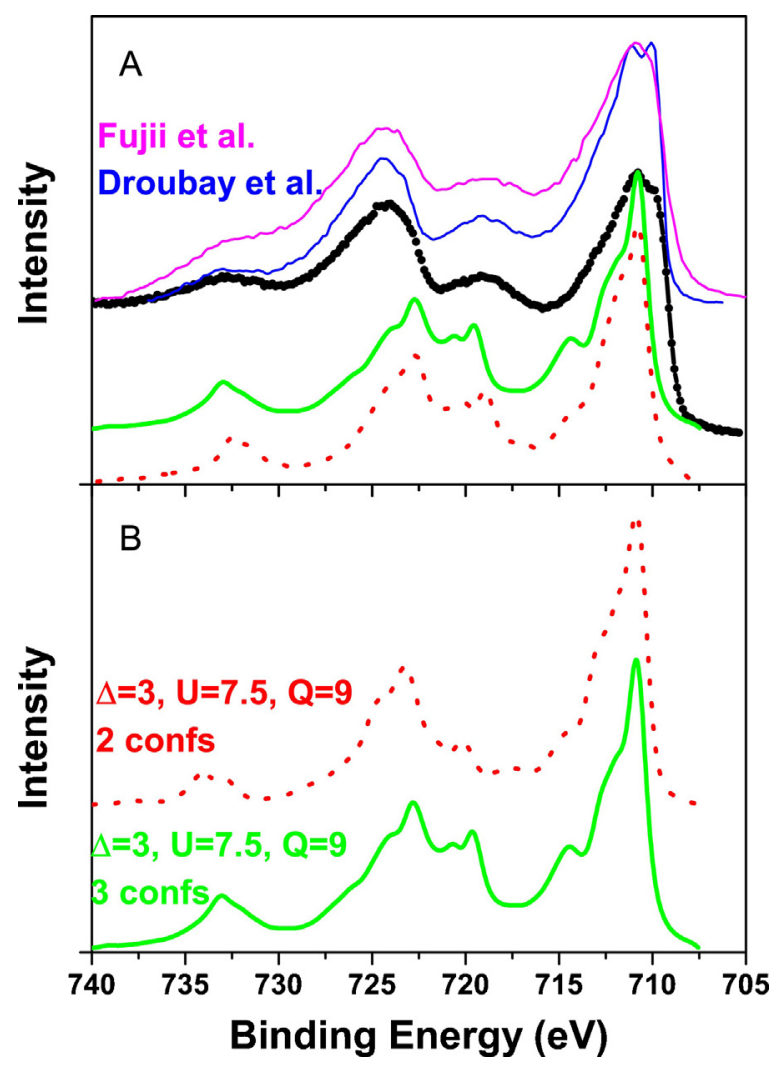

Fig. 3. (A) Experimental Fe $2 p$ photoemission of $\alpha-\mathrm{Fe}_{2} \mathrm{O}_{3}$ versus binding energy (black) compared with charge transfer multiplet calculations using charge transfer parameter set B [31] (red dotted) and set A (green solid). For comparison two reference 2p photoemission spectra as published by Fujii et al. [31] (pink) and Droubay et al. [34] (blue) are shown at the top. (B) Fe 2p photoemission charge transfer multiplet calculations with only one set of charge transfer parameters (set A, Table 1) with either three configurations (green solid, same as in panel A) or with two configurations (red dotted). (For interpretation of the references to color in this figure legend, the reader is referred to the web version of the article.)

symmetry at the surface is not taken into account in our multiplet calculations, which is the reason why the calculations do not entirely agree on this BE feature at $710 \mathrm{eV}$.

The $\mathrm{Fe} 2 \mathrm{p}$ photoemission simulations using our optimized charge transfer parameter sets $\mathrm{A}(\Delta=3, U=7.5$ and $Q=9.0$ and $10 \mathrm{Dq}=1.9 \mathrm{eV}$, Table 1 ) and $\mathrm{B}$ (from Fujii et al. [31], Table 1) are also compared with our Fe 2p HAXPES in Fig. 3A. The corresponding energies of the ground and final states of the parameter sets are written in Table 1 and show that the parameter set $B$ as used by Fujii et al. [31] and our optimized parameter set A have the same final state (EF1, EF2, EF3 and EF4) energies. Note that these relative state energies do not directly have a reference to the energy distances between peaks; compare for example with the relative energies of $2 \mathrm{p}_{1 / 2}, \mathrm{~S}$ and $\mathrm{S}^{\prime}(8.37,13.76$ and $22.5 \mathrm{eV})$ to $2 \mathrm{p}_{3 / 2}$. We also point out that the obtained parameters for $\Delta$ and $U$ of both set $A$ and $B$ relate well to estimates of Bocquet et al. for $\mathrm{Fe}^{3+}$ compounds [37]. The parameter set $A$ and $B$ differ in the fraction of the different states contributing to the ground state. For parameter set A, the contribution of the $3 \mathrm{~d}^{5}$ ionic state is increased.

Fig. 3B shows the difference between charge transfer multiplet calculations with three and two configurations using the charge transfer parameter set $\mathrm{A}$. The relatively small difference between the Fe $2 \mathrm{p}$ photoemission calculations with two or three configurations can be attributed to (i) the contribution of the third configuration is relatively small compared to the first two configurations and (ii) the third configuration in the $2 \mathrm{p}_{3 / 2}$ photoemission
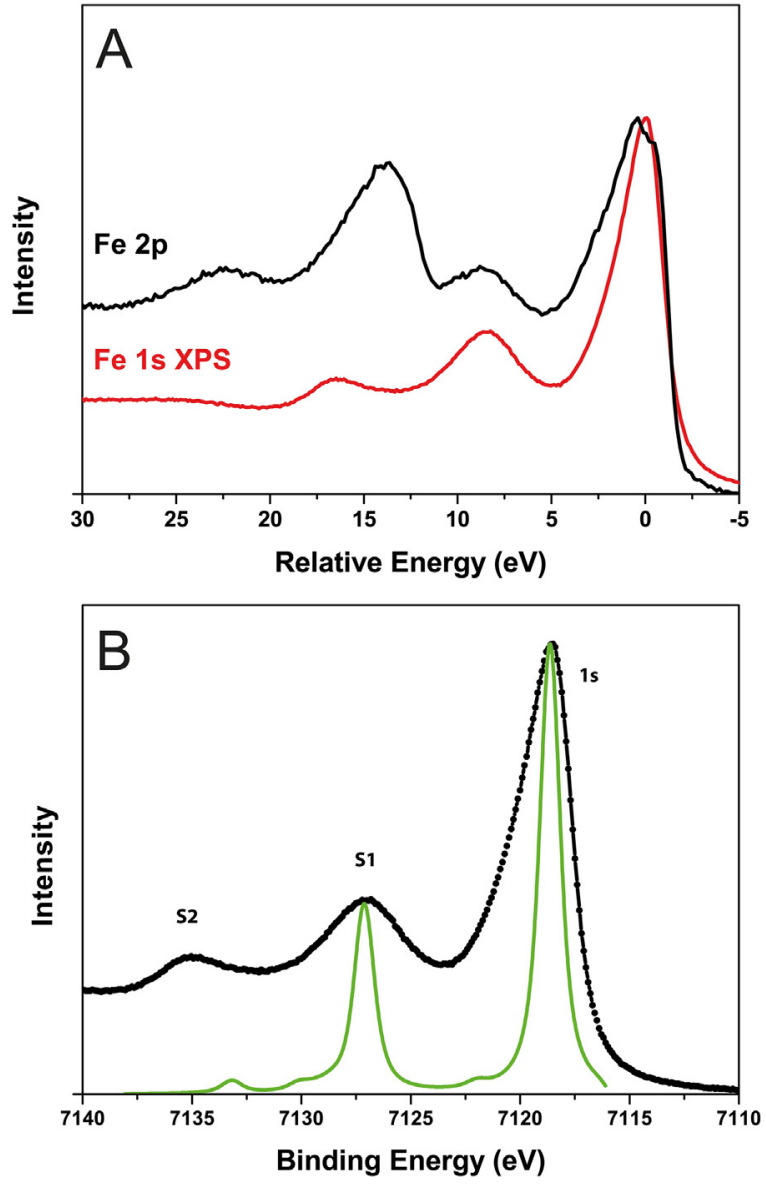

Fig. 4. (A) Experimental Fe 2p HAXPES (black) and Fe 1s HAXPES of $\alpha-\mathrm{Fe}_{2} \mathrm{O}_{3}$. The main Fe $1 \mathrm{~s}$ photoemission (red) and 2p photoemission (black) peak is shifted to zero. (B) Experimental Fe 1s HAXPES versus binding energy for $\alpha-\mathrm{Fe}_{2} \mathrm{O}_{3}$ (black) compared with charge transfer multiplet calculations using parameter set $\mathrm{A}$ (green solid). The charge transfer multiplet calculation was performed with $85 \%$ of the Hartree-Fock Slater integrals. (For interpretation of the references to color in this figure legend, the reader is referred to the web version of the article.)

will end up at about the $2 \mathrm{p}_{1 / 2}$ photoemission edge of the first configuration.

Fig. 4A shows the experimental Fe 2p HAXPES and the binding energy shifted $\mathrm{Fe} 1 \mathrm{~s}$ HAXPES of $\mathrm{Fe}_{2} \mathrm{O}_{3}$. The experimental $\mathrm{Fe} 1 \mathrm{~s}$ HAXPES shows three peaks. As mentioned above, on itself the creation of a photoelectron from the $1 \mathrm{~s}$ level should only give rise to a single peak, while the observation of three peaks suggests that at least three different states are contributing to the ground and final state of the process, namely the formal valence $3 d^{5}\left(\mathrm{Fe}^{3+}\right)$ and two additional states.

As displayed in Fig. 3B, it is not obvious from the Fe 2p photoemission that one should take into account three configurations, since the third configuration of $2 \mathrm{p}_{3 / 2}$ ends up below the $2 \mathrm{p}_{1 / 2}$ edge of the first configuration. Grosvenor et al. showed a multi-peak fitting of $\mathrm{Fe} 2 \mathrm{p}$ photoemission of $\mathrm{Fe}_{2} \mathrm{O}_{3}$ with three contributions and an extra surface contribution taken into account [30]. Three-state analysis was applied before on the $2 \mathrm{p}$ photoemission of the nickel and cobalt halides as well [33]. The comparison of Fe 1s HAXPES with 2p HAXPES in Fig. 4A shows that 2p3d multiplet effects and spin-orbit splitting disallow a direct observation in Fe 2p photoemission that two or three ionic configurations need to be taken into account, while it becomes clear directly from the Fe 1s HAXPES spectrum. This also means that it is very well possible that in many cases, where Fe $2 \mathrm{p}$ photoemission is analyzed in terms of charge 
transfer multiplet parameters, extra required configurations have been neglected.

Fig. 4B shows the comparison of the 1s experimental HAXPES with the calculated photoemission using charge transfer parameter set A. No background was subtracted from the experimental $1 \mathrm{~s}$ photoemission, which would lead to a substantial lower peak at $7135 \mathrm{eV}$ in the experimental spectrum. In addition, possibly a fourth configuration is required for the calculation in order to obtain an increase of the intensity of the peak at $7135 \mathrm{eV}$. This consideration is based on the relative energy difference between the EF4 and EF2 state (Table 1 ) that is around $10.5 \mathrm{eV}$. Note that both parameter set $A$ and $B$ show very similar Fe 1 s photoemission, but for parameter set $A$, the one that is shown in Fig. 4B, the peak around $7127 \mathrm{eV}$ agrees slightly better with the energy of the experimentally observed peak in this range and the feature at lowest BE is at slightly lower energy for the third band at $7135 \mathrm{eV}$ compared to parameter set $\mathrm{B}$.

The charge transfer multiplet program uses $\Delta, U$ and $Q$, which we have made general for $1 \mathrm{~s}$ and $2 \mathrm{p}$ photoemission. Especially for $\Delta$ and $U$ this is logical, since there is no difference between $U$ in 1 s or 2 p photoemission, since $U$ comes from $3 \mathrm{~d}-3 \mathrm{~d}$ interaction and there is no reason to assume that the relative energies of the ionic configurations, determined by $\Delta$ and $U$ in the initial state would be different for $2 \mathrm{p}$ and $1 \mathrm{~s}$ photoemission. In addition, we assumed that the core hole potential is the same for a $2 \mathrm{p}$ and 1 s hole, which leads to the same $Q$ in simulations of $2 \mathrm{p}$ and $1 \mathrm{~s}$ photoemission.

In reference [16] Karis et al. reported that the 3d shake-up for $\mathrm{Ni}$ metal is found at different relative energies for the $1 \mathrm{~s}$ photoemission ( $4 \mathrm{eV}$ ) and the $2 \mathrm{p}_{3 / 2}$ photoemission $(6 \mathrm{eV})$ compared to the main photoemission line. We claimed above that $\Delta, U$ and $Q$ can be taken general for $1 \mathrm{~s}$ and $2 \mathrm{p}$ photoemission. In addition, we want to point out that as well the energies of the different configurations in the ground state (EGX) and the crystal field 10Dq do not depend on the core hole (either $1 \mathrm{~s}$ and $2 \mathrm{p}$ ) created in the same sample (but depend on the sample). Following this reasoning, the energies of the configurations in the final state (EFx) could also not be responsible for the observation in [16] since those depend directly on the $\Delta, U$ and $Q$. Because the direct and exchange Coulomb interactions between $2 \mathrm{p}$ and $3 \mathrm{~d}$ electrons are much stronger than between $1 \mathrm{~s}$ and $3 \mathrm{~d}$ electrons and (in addition there is a $2 \mathrm{p}$ core hole splitting compared to no splitting for $1 \mathrm{~s}$ core holes) and because of this difference in electron interaction strength, we believe that different relative energies of shake-up features compared to the main photoemission line can be expected. By coincidence the first shake-up for Fe $1 \mathrm{~s}$ photoemission $(8.57 \mathrm{eV}$ ) compared to Fe $2 \mathrm{p}$ photoemission $(8.37 \mathrm{eV})$ of $\mathrm{Fe}_{2} \mathrm{O}_{3}$ is almost at the same relative energy, but this is not necessarily the case as was observed in [16]. Note that in their analysis, Karis et al. changed the value of $Q$ ( or $Q-\Delta$ as they mention) slightly and changed as well the $U$ between 1 s and $2 \mathrm{p}$ photoemission (where we believe $U$ should not depend on the $1 \mathrm{~s}$ or $2 \mathrm{p}$ hole). As well, while Karis et al. observe that the Fe $2 \mathrm{p}_{3 / 2}$ photoemission shake-up is at $6 \mathrm{eV}$, their simulation shows that there is a small contribution already at about $4 \mathrm{eV}$. It is just that the multiplets resulting from the $2 \mathrm{p} 3 \mathrm{~d}$ electron interaction lead to a higher intensity contribution at $6 \mathrm{eV}$. Another interesting observation they make is that the shake-up of $2 \mathrm{p}_{1 / 2}$ is at $4.6 \mathrm{eV}$, which is still a little bit higher than the shake-up of $1 \mathrm{~s}$ photoemission. This would imply that the $2 \mathrm{p}_{1 / 2} 3 \mathrm{~d}$ electron interaction is already much weaker than $2 \mathrm{p}_{3 / 2} 3 \mathrm{~d}$, an observation which fits well to features obtained in the $\mathrm{L}_{3}$ versus $\mathrm{L}_{2}$ edge of $\mathrm{X}$-ray absorption $[5,6,25]$.

In summary, one can conclude that $1 \mathrm{~s}$ photoemission is the best reference experiment to perform to obtain the relevant charge transfer parameters. We point out that the extra ionic configurations are not of relevance for X-ray absorption analysis as has been pointed out before [38].

\section{Summary and conclusions}

We have shown both Fe 2p and 1s HAXPES of $\mathrm{Fe}_{2} \mathrm{O}_{3}$. Although not mentioned before we would like to state that the presented charge transfer parameters obtained for Fe $2 \mathrm{p}$ and $1 \mathrm{~s}$ HAXPES could also be applied on calculations of the $\mathrm{Fe} 3 \mathrm{~s}$ and $3 \mathrm{p}$ photoemission. The values obtained from the calculated Fe $2 \mathrm{p}$ and 1s HAXPES of $\alpha-\mathrm{Fe}_{2} \mathrm{O}_{3}$ are in close agreement with values found for Fe $2 \mathrm{p}$ photoemission by Fujii et al. [31] and Bocquet et al. [37]. The Fe 1s HAXPES contains three distinct features and this directly implies that at least three configurations have to be taken into account in the charge transfer multiplet calculations of Fe photoemission of $\mathrm{Fe}_{2} \mathrm{O}_{3}$. On the other hand, there is not a big difference between two and three configuration in the case of Fe $2 \mathrm{p}$ photoemission due to the multiplet and $2 \mathrm{p}$ spin orbit coupling complicating the direct observation of a third configuration. Overall, the Fe 1s HAXPES gives a more direct way to obtain the charge transfer parameters in the charge transfer multiplet photoemission calculations than Fe 2p photoemission and 1s HAXPES shows clearly the amount of states that have to be taken into consideration in theoretical treatment.

\section{Acknowledgements}

ESRF is acknowledged for provision of beam time under the experiment number HE-3459. The scientist team from ID16, and especially Roberto Verbeni, is acknowledged for support during the beamtime. ID08 is thanked for the supply of the $\alpha-\mathrm{Fe}_{2} \mathrm{O}_{3}$ crystal sample. Zoran Ristanovic is acknowledged for help during the beamtime. This work was partially funded by the Italian Ministry of Research through the project PRIN 'Interfacce di ossidi: nuove proprietà emergenti, multifunzionalità e dispositivi per l'elettronica e l'energia (OXIDE).

\section{References}

[1] W. Drube (Ed.), Recent advances in hard X-ray photoelectron spectroscopy, J. Electron Spectrosc. Relat. Phenom. 190 (2013) 125-314, http://dx.doi.org/10. 1016/j.elspec.2013.11.006

[2] F. Reinert, S. Hüfner, Photoemission spectroscopy-from early days to recent applications, N. J. Phys. 7 (2005) 97, http://dx.doi.org/10.1088/1367-2630/7/1/ 097

[3] J. Zaanen, C. Westra, G.A. Sawatzky, Determination of the electronic structure of transition-metal compounds: 2p X-ray photoemission spectroscopy of the nickel dihalides, Phys. Rev. B 33 (1986) 8060-8073.

[4] T.A. Carlson, J.C. Carver, G.A. Vernon, Satellite Structure in the X-ray photoelectron spectra of the K shell of transition metal compounds, J. Chem. Phys. 62 (1975) 932-935.

[5] F. de Groot, A. Kotani, Core Level Spectroscopy of Solids, 2008

[6] P.S. Miedema, M.M. van Schooneveld, R. Bogerd, T.C.R. Rocha, M. Hävecker A. Knop-Gericke, et al., Oxygen binding to cobalt and iron phthalocyanines as determined from in situ X-ray absorption spectroscopy, J. Phys. Chem. C 115 (2011) 25422-25428, http://dx.doi.org/10.1021/jp209295f

[7] G. Beamson, S.R. Haines, N. Moslemzadeh, P. Tsakiropoulos, J.F. Watts, P. Weightman, et al., Performance and application of a high energy monochromated $\mathrm{Cu} K \alpha 1$ X-ray source for the electron spectroscopy of materials, J. Electron Spectrosc. Relat. Phenom. 142 (2005) 151-162, http://dx.doi.org/10. 1016/j.elspec.2004.10.008

[8] G. Beamson, S.R. Haines, N. Moslemzadeh, P. Tsakiropoulos, P. Weightman, J.F. Watts, High-energy monochromated $\mathrm{Cu} K \alpha 1$ X-ray source for electron spectroscopy of materials: initial results, Surf. Interface Anal. 36 (2004) 275-279, http://dx.doi.org/10.1002/sia.1744

[9] M. Kobata, I. Pís, H. Iwai, H. Yamazui, H. Takahashi, M. Suzuki, et al., Development of the hard-X-ray angle resolved X-ray photoemission spectrometer for laboratory use, Anal. Sci. 26 (2010) 227-232, http://dx.doi.org/10.2116/analsci. 26.227

[10] P. Le Fevre, H. Magnan, D. Chandesris, J. Jupille, S. Bourgeois, A. Barbier, et al, Hard X-ray resonant electronic spectroscopy in transition metal oxides, Nucl. Instrum. Methods Phys. Res. Sect. A 547 (2005) 176-186.

[11] A. Némethy, L. Kövér, I. Cserny, D. Varga, P.B. Barna, The KLL and KLM Auge spectra of 3d transition metals, $Z=23-26$, J. Electron Spectrosc. Relat. Phenom. 82 (1996) 31-40, http://dx.doi.org/10.1016/S0368-2048(96)03038-1

[12] L. Kövér, Z. Berényi, I. Cserny, L. Lugosi, W. Drube, T. Mukoyama, et al., Initialand final-state excitations in K L23 L23 Auger spectra of $\mathrm{Cu}$ and Ni metals, induced near threshold, Phys, Rev. B - Condens. Matter Mater. Phys. 73 (2006) 195101, http://dx.doi.org/10.1103/PhysRevB.73.195101 
[13] N. Moslemzadeh, G. Beamson, P. Tsakiropoulos, J.F. Watts, Investigation of intrinsic plasmon energy losses associated with the Fe $1 \mathrm{~s}$ core level in metallic iron, Surf. Sci. 600 (2006) 265-272, http://dx.doi.org/10.1016/j.susc.2005.10. 049

[14] N. Moslemzadeh, G. Beamson, S.R. Haines, P. Tsakiropoulos, J.F. Watts, P. Weightman, High-energy X-ray photoelectron spectroscopy with new monochromatised Cu Ka1 X-rays; characteristics, capabilities and limitations, Surf. Interface Anal. 38 (2006) 703-706, http://dx.doi.org/10.1002/sia

[15] N. Moslemzadeh, G. Beamson, P. Tsakiropoulos, J.F. Watts, S.R. Haines, P. Weightman, Electronic structure of Ti metal and $\mathrm{TiO}_{2}$ powder studied by hard and soft $(\mathrm{Cu} \mathrm{K} \alpha 1$ and $\mathrm{Al} \mathrm{K} \alpha 1)$ X-ray photoelectron and Auger spectroscopy, J. Electron Spectrosc. Relat. Phenom. 152 (2006) 148-151, http://dx.doi.org/10. 1016/j.elspec.2006.05.003

[16] O. Karis, S. Svensson, J. Rusz, P.M. Oppeneer, M. Gorgoi, F. Schäfers, et al., Highkinetic-energy photoemission spectroscopy of $\mathrm{Ni}$ at $1 \mathrm{~s}$ : $6-\mathrm{eV}$ satellite at $4 \mathrm{eV}$ Phys. Rev. B 78 (2008) 233105.

[17] M. Calandra, J.P. Rueff, C. Gougoussis, D. Céolin, M. Gorgoi, S. Benedetti, et al., K-edge X-ray absorption spectra in transition-metal oxides beyond the singleparticle approximation: Shake-up many-body effects, Phys. Rev. B 86 (2012) 165102, http://dx.doi.org/10.1103/PhysRevB.86.165102

[18] I. Vobornik, G. Panaccione, J. Fujii, Z.H. Zhu, F. Offi, B.R. Salles, et al., Observation of distinct bulk and surface chemical environments in a topological insulator under magnetic doping, J. Phys. Chem. C 118 (2014) 12333-12339, http://dx. doi.org/10.1021/jp502729u

[19] P.S. Miedema, S. Stepanow, P. Gambardella, F.M.F. De Groot, 2p X-ray absorption of iron-phthalocyanine, J. Phys. Conf. Ser. 190 (2009) 012143, http://dx.doi.org/ 10.1088/1742-6596/190/1/012143

[20] S. Tanuma, C.J. Powell, D.R. Penn, Calculations of electron inelastic mean free paths, Surf. Interface Anal. 20 (1993) 77-89, http://dx.doi.org/10.1002/sia. 1997

[21] M. Sacchi, F. Offi, P. Torelli, A. Fondacaro, C. Spezzani, M. Cautero, et al. Quantifying the effective attenuation length in high-energy photoemission experiments, Phys. Rev. B 71 (2005) 155117

[22] G. Paolicelli, A. Fondacaro, F. Offi, G. Stefani, G. Cautero, M. Cautero, et al. Bulk sensitive photoemission: first results of VOLPE project at ESRF, J. Electron Spectrosc. Relat. Phenom. 144-147 (2005) 963-966, http://dx.doi.org/10. 1016/j.elspec.2005.01.114

[23] P. Torelli, M. Sacchi, G. Cautero, M. Cautero, B. Krastanov, P. Lacovig, et al., Experimental setup for high energy photoemission using synchrotron radiation, Rev. Sci. Instrum. 76 (2005) 023909, http://dx.doi.org/10.1063/1.1852323

[24] R.D. Cowan, The Theory of Atomic Structure and Spectra, University of California Press, Berkeley/Los Angeles, CA, 1981
[25] F.M.F. de Groot, J.C. Fuggle, B.T. Thole, G.A. Sawatzky, 2p X-ray absorption of 3d transition-metal compounds: an atomic multiplet description including the crystal field, Phys. Rev. B 42 (1990) 5459-5468.

[26] G. van der Laan, J. Zaanen, G.A. Sawatzky, R. Karnatak, J.-M. Esteva, Comparison of X-ray absorption with X-ray photoemission of nickel dihalides and NiO, Phys. Rev. B 33 (1986) 4253-4263.

[27] G. van der Laan, J. Zaanen, G.A. Sawatzky, R. Karnatak, J.-M. Esteva, Correlation effects, charge-transfer energies and covalency in nickel compounds as determined by X-ray absorption spectroscopy, Solid State Commun. 56 (1985) 673-676.

[28] L.F. Mattheiss, R.E. Dietz, Relativistic tight-binding calculation of core-valence transitions in Pt and Au, Phys. Rev. B 22 (1980) 1663-1676.

[29] P. Mills, J.L. Sullivan, A study of the core level electrons in iron and its three oxides by means of X-ray photoelectron spectroscopy, J. Phys. D: Appl. Phys. 16 (1983) 723-732.

[30] A.P. Grosvenor, B.A. Kobe, M.C. Biesinger, N.S. McIntyre, Investigation of multiplet splitting of Fe 2 p XPS spectra and bonding in iron compounds, Surf. Interface Anal. 36 (2004) 1564-1574.

[31] T. Fujii, F.M.F. de Groot, G.A. Sawatzky, F.C. Voogt, T. Hibma, K. Okada, In situ XPS analysis of various iron oxide films grown by $\mathrm{NO}_{2}$-assisted molecular-beam epitaxy, Phys. Rev. B 59 (1999) 3195-3202.

[32] P.S. Miedema, F.M.F. de Groot, The iron L edges: Fe 2p X-ray absorption and electron energy loss spectroscopy, J. Electron Spectrosc. Relat. Phenom. 187 (2013) 32-48, http://dx.doi.org/10.1016/j.elspec.2013.03.005

[33] K. Okada, A. Kotani, B.T. Thole, Charge transfer satellites and multiplet splitting in X-ray photoemission spectra of late transition metal halides, J. Electron Spectrosc. Relat. Phenom. 58 (1992) 325-343, http://dx.doi.org/10.1016/03682048(92)85018-3

[34] T. Droubay, S. Chambers, Surface-sensitive Fe $2 p$ photoemission spectra for $\alpha$ $\mathrm{Fe}_{2} \mathrm{O}_{3}(0001)$ : the influence of symmetry and crystal-field strength, Phys. Rev. B 64 (2001) 205414, http://dx.doi.org/10.1103/PhysRevB.64.205414

[35] A. Fujimori, M. Saeki, N. Kimizuka, M. Taniguchi, S. Suga, Photoemission satellites and electronic structure of $\mathrm{Fe}_{2} \mathrm{O}_{3}$, Phys. Rev. B 34 (1986) 7318-7328.

[36] R. Zimmermann, P. Steiner, R. Claessen, F. Reinert, S. Hüfner, P. Blaha, et al. Electronic structure of 3d-transition-metal oxides: on-site Coulomb repulsion versus covalency, J. Phys.: Condens. Matter 11 (1999) 1657-1682.

[37] A.E. Bocquet, T. Mizokawa, K. Morikawa, A. Fujimori, S. Barman, K. Maiti, et al., Electronic structure of early 3d-transition-metal oxides by analysis of the $2 \mathrm{p}$ core-level photoemission spectra, Phys. Rev. B: Condens. Matter 53 (1996) 1161-1170, http://www.ncbi.nlm.nih.gov/pubmed/9983572

[38] F. de Groot, Multiplet effects in X-ray spectroscopy, Coord. Chem. Rev. 249 (2005) 31-63, http://dx.doi.org/10.1016/j.ccr.2004.03.018. 